\title{
Random Rotation-based Low-Complexity Schemes for Intelligent Reflecting Surfaces
}

\author{
Constantinos Psomas, Ilias Chrysovergis, and Ioannis Krikidis \\ IRIDA Research Centre for Communication Technologies \\ Department of Electrical and Computer Engineering, University of Cyprus, Cyprus \\ Email: \{psomas, chrysovergis.ilias, krikidis\}@ucy.ac.cy
}

\begin{abstract}
The employment of intelligent reflecting surfaces (IRSs) is a potential solution to increase the spectral and energy efficiency of wireless networks. The passive operation of their elements and the fact they can be deployed on any flat surface, makes them ideal for future wireless networks. However, the passive operation of their elements, incurs limitation on their capabilities. In this paper, we propose two low-complexity and energy efficient techniques for IRS-aided communications, namely, a coding-based and a selection-based scheme, both based on random phase rotations. In particular, the coding-based scheme uses time-varying random phase rotations to produce a timevarying channel. The selection-based scheme, selects and activates a partition of the IRS elements at each time slot based on the received signal power at the destination. Analytical expressions for both schemes are derived and the achieved performance gains are demonstrated. Furthermore, it is shown that both schemes provide full diversity order.
\end{abstract}

Index Terms-Intelligent reflecting surfaces, outage probability, energy efficiency, selection, diversity.

\section{INTRODUCTION}

Even though the 5G-era has commenced, with its deployment in some countries, the challenge of how to connect billions of devices and satisfy their rate requirements still exists. Moreover, the energy efficient of such highly dense and highly connected wireless networks is another vital requirement. A promising new technology to address these issues is the so-called intelligent reflecting surface (IRS) [1]. An IRS consists of an array of passive elements embedded in a flat surface, where each element is reconfigurable and can alter the phase of the incident signal through a dedicated controller. Their employment can provide many benefits such as extend the range of wireless systems, improve spectral efficiency due to their full-duplex operation but also increase energy efficiency due to the passive operation of their elements.

The consideration of IRSs in wireless networks has recently appeared in the literature in various different scenarios [2]-[9]. In [2], the authors study a single cell wireless system where a multi-antenna access point (AP) communicates with multiple users via an IRS; it is verified that by jointly optimizing the

This work was co-funded by the European Regional Development Fund and the Republic of Cyprus through the Research and Innovation Foundation, under the project INFRASTRUCTURES/1216/0017 (IRIDA). It has also received funding from the European Union's Horizon 2020 research and innovation programme under grant agreement No 812991.

978-1-7281-4490-0/20/\$31.00 @ 2020 IEEE active beamforming from the AP and the passive beamforming from the IRS, provides performance gains. A similar scenario is considered in [3], where it is shown that massive multipleinput multiple output (MIMO)-like gains can be achieved with a smaller number of antennas at the AP. The implementation of two index modulation schemes, space shift keying and spatial modulation, in IRS-aided communications is studied in [4]. It is shown that good spectral efficiency performance can be achieved even for low signal-to-noise ratio (SNR) values. The authors in [5], consider IRS-assisted non-orthogonal multiple access (NOMA) communications and it is demonstrated that NOMA can benefit from the employment of IRSs.

The energy efficiency of IRS deployments was investigated in [6], where the proposed resource allocation methods achieved up to $300 \%$ higher energy efficiency compared to the conventional multi-antenna amplify-and-forward relaying. A stochastic geometry model with IRSs is presented in [7], where the spatial randomness of the users is taken into account. The derived analytical framework for the spectral and energy efficiency of the proposed model validates the gains from the employment of multiple IRSs. The implementation of IRSs has also been considered in the context of simultaneous wireless information and power transfer [8], [9]. Specifically, the work in [8], considers an IRS-aided wireless system with multiple information receivers and energy harvesters. By maximizing the weighted sum-power at the energy harvesters, it is demonstrated that IRS can enhance the performance. A similar scenario is considered in [9], where by maximizing the weighted sum-rate of the information receivers under energy harvesting constraints, it is shown that the employment of IRS benefits the network.

Most of the aforementioned works, focus on optimizing the incident signal's phase shifts at the IRS and assume knowledge of the channel state information. However, this corresponds to higher complexity and power consumption but can also be impractical in some cases. Motivated by this, in this paper, we propose two low-complexity and energy efficient schemes for IRS-aided communications via random phase rotations. Specifically, we propose a coding-based scheme, inspired by the rotate-and-forward protocol [10], which produces a timevarying channel through time-varying random rotations. Furthermore, we propose a selection-based scheme, which activates a partition of the IRS elements based on the highest received signal power. The performance metrics of interest are the outage 
probability and energy efficiency, for which we derive analytical expressions for both schemes. Our results demonstrate that the proposed schemes provide significant performance gains compared to the conventional case. In addition, we show that the coding-based scheme exhibits full time diversity order, whereas the selection-based scheme provides full spatial diversity order.

\section{SySTEM MODEL}

Consider an IRS-aided network, where a source $S$ achieves communications with a destination $D$ through the employment of an IRS with $M$ reflecting elements, as shown in Fig. 1. The source and destination are equipped with a single antenna and a direct link between them is not available (e.g., due to obstacles). A codeword $\mathbf{X} \triangleq\left[x_{1}, x_{2}, \ldots, x_{T}\right], x_{t} \in \mathbb{C}, 1 \leq$ $t \leq T$, is transmitted by the source over $T$ symbols time; we assume that $T<M$. All wireless links are assumed to exhibit Rayleigh fading; we define by $h_{i}$ and $g_{i}$ the fading coefficients from $S$ to the $i$-th element and from the $i$-th element to $D$, respectively. The fading coefficients remain constant during the $T$ transmissions but change independently every $T$ channel uses according to a complex Gaussian distribution with zero mean and unit variance, i.e. $h_{i} \sim \mathcal{C N}(0,1)$ and $g_{i} \sim \mathcal{C N}(0,1)$.

We assume that knowledge of any channel state information does not exist. At every time instant $t$, each element of the IRS, randomly rotates (shifts) the phase of the incident signal. Let

$$
\boldsymbol{\Phi}_{t}=\operatorname{diag}\left[\exp \left(\jmath \phi_{t, 1}\right) \exp \left(\jmath \phi_{t, 2}\right) \ldots \exp \left(\jmath \phi_{t, M}\right)\right]
$$

be the diagonal matrix containing the independent and identically distributed phase shift variables. Due to the random rotations, the variables $\phi_{t, i}$ are uniformly distributed in $[0,2 \pi)$. Therefore, if the source transmits with a constant power $P$, the received signal at the destination $D$ at the $t$-th channel use can be written as

$$
r_{t}=\sqrt{P} \mathbf{h}^{T} \boldsymbol{\Phi}_{t} \mathbf{g} x_{t}+n_{t}
$$

where $\mathbf{h}=\left[\begin{array}{llll}h_{1} & h_{2} & \ldots & h_{M}\end{array}\right]^{T}, \mathbf{g}=\left[\begin{array}{llll}g_{1} & g_{2} & \ldots & g_{M}\end{array}\right]^{T}$, and $n_{t} \sim$ $\mathcal{C N}\left(0, \sigma^{2}\right)$ is the additive white Gaussian noise with variance $\sigma^{2}$. Then, the instantaneous SNR at the destination $D$ over the $t$-th transmission is

$$
\gamma_{t}=\frac{P}{\sigma^{2}} H_{t}
$$

where

$$
H_{t}=\left|\sum_{i=1}^{M} h_{i} g_{i} \exp \left(\jmath \phi_{t, i}\right)\right|^{2}
$$

is the channel gain from the $M$ elements of the IRS.

\section{RANDOM ROTATION-BASED IRS SCHEMES}

In this section, we describe our proposed low-complexity schemes and derive analytical expressions for the achieved outage probability,

$$
\Pi(\rho, T)=\mathbb{P}\left\{\frac{1}{T} \sum_{t=1}^{T} \log _{2}\left(1+\gamma_{t}\right)<\rho\right\},
$$

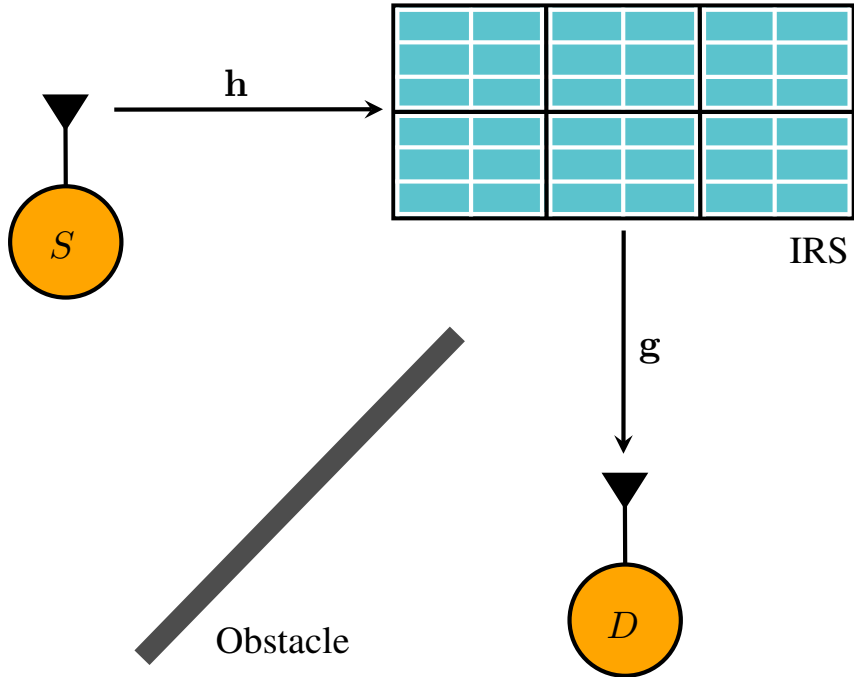

Fig. 1. The considered IRS-aided communication network.

where $\rho$ is a non-negative pre-defined threshold, and the endto-end energy efficiency,

$$
\eta=\frac{\mathbb{E}\left\{\frac{1}{T} \sum_{t=1}^{T} \log _{2}\left(1+\gamma_{t}\right)\right\}}{P_{c}},
$$

where $P_{c}$ is the system's total power consumption.

Before we proceed, we state the proposition below for the conventional case $T=1$, which will be used as a benchmark for both schemes.

Proposition 1. The outage probability achieved by a random rotation for $T=1$ is given by

$$
\Pi(\rho, 1)=1-\frac{2}{\Gamma(M)}\left(\frac{\theta \sigma^{2}}{P}\right)^{\frac{M}{2}} K_{M}\left(2 \sqrt{\frac{\theta \sigma^{2}}{P}}\right),
$$

where $\theta \triangleq 2^{\rho}-1$.

Proof. See Appendix A.

\section{A. Coding-based scheme}

In the coding-based scheme, the destination receives the superposition of $M$ independent channels at each time instant $t$. The different phase shifts induced by the IRS elements at each time slot introduce an artificial fast fading channel, which can increase the performance [10], without any knowledge regarding the channel at the source, the IRS or the destination. Note that the instantaneous SNRs between different time slots are correlated. As this makes the derivation of the outage probability challenging, we present two approximations in the following two theorems. In particular,

- Theorem 1 provides an approximation by assuming channel gains $H_{t}$ are mutually independent.

- Theorem 2 employs the central limit theorem (CLT) and approximates $H_{t}$ as exponential random variables.

We show that both approximations are sufficient and appropriate to describe the proposed scheme's behavior. 
Theorem 1. The outage probability of the coding-based scheme, under the independence assumption, is approximated by

$$
\begin{aligned}
\Pi_{\mathrm{CB}}^{\mathrm{IND}}(\rho, T) \approx( & \left.\frac{\sigma^{2}}{P}\right)^{T-1} \int_{1}^{\xi_{T}} \cdots \int_{1}^{\xi_{2}}\left(1-\frac{2 \Theta^{M / 2}}{\Gamma(M)} K_{M}(2 \sqrt{\Theta})\right) \\
& \times \prod_{t=2}^{T} f_{H}\left(\frac{\sigma^{2}}{P}\left(w_{t}-1\right)\right) d w_{2} \cdots d w_{T}
\end{aligned}
$$

where $\xi_{i}=2^{\rho T} / \prod_{t=i+1}^{T} w_{t}, 2 \leq i \leq T$,

$$
\Theta \triangleq \frac{\sigma^{2}}{P}\left(\frac{2^{\rho T}}{\prod_{t=2}^{T} w_{t}}-1\right)
$$

and

$$
f_{H}(h)=\frac{2 h^{(M-1) / 2} K_{M-1}(2 \sqrt{h})}{\Gamma(M)} .
$$

Proof. See Appendix B.

It is clear that for the case $T=1$, Theorem 1 corresponds to the exact analytical result in Proposition 1. Next, we derive the outage probability with the use of the CLT.

Theorem 2. The outage probability of the coding-based scheme, under the CLT, is approximated by

$$
\begin{aligned}
\Pi_{\mathrm{CB}}^{\mathrm{CLT}}(\rho, T) \approx & \left(\frac{\sigma^{2}}{M P}\right)^{T-1} \int_{1}^{\xi_{T}} \cdots \int_{1}^{\xi_{2}}\left(1-\exp \left(-\frac{\Theta}{M}\right)\right) \\
& \times \prod_{t=2}^{T} \exp \left(-\frac{\sigma^{2}}{M P}\left(w_{t}-1\right)\right) d w_{2} \cdots d w_{T}
\end{aligned}
$$

where $\Theta$ and $\xi_{i}$ are given in Theorem 1.

Proof. See Appendix C.

Remark 1. Even though the theorems above are derived based on the assumption that $M$ is large, they also work well for small M. Overall, Theorem 1 provides the best approximation for any value of $M$. On the other hand, Theorem 2, due to its simplest mathematical form, can provide further system insights by assisting in the derivation of the diversity order and coding gain (see below). Finally, asymptotically $(M \rightarrow \infty)$, both theorems produce the same results.

Since the correlation between the random variables $H_{t}$ decreases with $M$ (see Appendix B and Fig. 5), the codingbased scheme starts to exhibit diversity gains. To derive the diversity order and coding gain for the coding-based scheme, we need to consider $P \rightarrow \infty$. By using the approximation $K_{M}(x) \approx \Gamma(M) 2^{M-1} / x^{M}$ for $x \approx 0$ [11] in Theorem 1 , we can see that the outage probability $\Pi_{\mathrm{CB}}^{\mathrm{IND}}(\rho, T)$ reduces to zero. This is because the convergence to the diversity order for cascaded channels is very slow and is observed for very high SNR values [12]. However, using the approximated expression in Theorem 2, we have

$$
\lim _{P \rightarrow \infty} \Pi_{\mathrm{CB}}^{\mathrm{CLT}}(\rho, T) \approx\left(\frac{\sigma^{2}}{M P}\right)^{T} \int_{1}^{\xi_{T}} \cdots \int_{1}^{\xi_{2}}\left(\frac{2^{\rho T}}{\prod_{t=2}^{T} w_{t}}-1\right)
$$

$$
\begin{aligned}
& \times \prod_{t=2}^{T}\left(1-\frac{\sigma^{2}}{M P}\left(w_{t}-1\right)\right) d w_{2} \cdots d w_{T} \\
& \rightarrow O\left(1 / P^{T}\right),
\end{aligned}
$$

where it is clear that the expansion of the second product in (12), will be a sum of $2^{T-1}$ terms, out of which only the term equal to one will not contain $1 / P$. Therefore, as the smallest order term will dominate the others, it follows that the codingbased scheme achieves time diversity of order $T$ with coding gain [13] equal to

$$
\begin{gathered}
G_{\mathrm{CB}}=\left(\frac{\sigma^{2}}{M}\right)^{T} \int_{1}^{\xi_{T}} \cdots \int_{1}^{\xi_{2}}\left(\frac{2^{\rho T}}{\prod_{t=2}^{T} w_{t}}-1\right) d w_{2} \cdots d w_{T} \\
=\left(\frac{\sigma^{2}}{M}\right)^{T}(-1)^{T}\left(1-2^{\rho T} \sum_{t=0}^{T-1} \frac{(-1)^{t}}{t !} \log ^{t}\left(2^{\rho T}\right)\right)
\end{gathered}
$$

which follows by evaluating the $(T-1)$-fold integral and after some trivial algebraic manipulations.

We now turn our attention to the energy efficiency achieved by this scheme, as described by (6), which takes into account the expected rate of the scheme. Note that the expected rate is independent of $T$ since $\mathbb{E}\left\{\frac{1}{T} \sum_{t=1}^{T} \log _{2}\left(1+\gamma_{t}\right)\right\}=$ $\frac{1}{T} \sum_{t=1}^{T} \mathbb{E}\left\{\log _{2}\left(1+\gamma_{t}\right)\right\}=\mathbb{E}\left\{\log _{2}\left(1+\gamma_{t}\right)\right\}$. Then, we can state the following.

Proposition 2. The expected rate achieved by the coding-based scheme is

$$
R_{\mathrm{CB}}=\frac{2}{\Gamma(M)} \int_{0}^{\infty}\left(\frac{\theta \sigma^{2}}{P}\right)^{\frac{M}{2}} K_{M}\left(2 \sqrt{\frac{\theta \sigma^{2}}{P}}\right) d \rho,
$$

where $\theta \triangleq 2^{\rho}-1$.

Proof. The result follows simply from the fact that the expectation of a non-negative random variable $X$ is given by $\mathbb{E}\{X\}=\int_{x>0} \mathbb{P}\{X>x\} d x$. The final expression is derived by the use of Proposition 1.

Then, from (6), we have that the energy efficiency achieved by the coding-based scheme is

$$
\eta_{\mathrm{CB}}=\frac{R_{\mathrm{CB}}}{P / \xi+P_{S}+P_{D}+P_{\mathrm{IRS}}},
$$

where $\xi$ is the amplifier's efficiency, whereas $P_{S}, P_{D}$ and $P_{\text {IRS }}$ is the static power consumption at the source, destination and IRS, respectively [6]. The power consumption at the IRS depends on the number of activated elements, that is, $P_{\mathrm{IRS}}=M P_{E}$, where $P_{E}$ is the power consumed to operate a single element.

\section{B. Selection-based scheme}

For the selection-based scheme, we consider $T=1$ and assume that the IRS is partitioned into $N$ non-overlapping sub-surfaces of $m$ elements, where $N$ is a divisor of $M$, i.e. $m N=M$. An example of the system model is illustrated in Fig. 1 with $N=6$ and $m=6$, where the partitions are shown 
by the solid lines. We assume there is knowledge of the received SNR power at the destination from each sub-surface; this can be implemented by a training period, where the received signal strength indicator (RSSI) is fed back to the IRS controller. Then, at each time slot, the IRS controller selects and activates the sub-surface which achieves the highest SNR at the destination.

The outage probability achieved by the proposed selectionbased scheme is given below.

Proposition 3. The outage probability of the selection-based scheme is $\Pi_{\mathrm{SB}}(\rho)=\Pi(\rho, 1)^{N}$, where $N$ is the number of subsurfaces with $m$ elements and $\Pi(\rho, 1)$ is the outage probability of a random selection given by Proposition 1 with $M=m$.

Proof. Assume the ordering $\gamma_{(1)} \geq \gamma_{(2)} \geq \cdots \geq \gamma_{(N)}$, where $\gamma_{(i)}, 1 \leq i \leq N$, is the $i$-th highest receiver SNR at the destination from the $N$ sub-surfaces of the IRS. Then, using the distribution of ordered random variables, the outage probability is [14]

$$
\Pi_{\mathrm{SB}}(\rho)=\mathbb{P}\left\{\log _{2}\left(1+\gamma_{1}\right)<\rho\right\}^{N},
$$

where $\mathbb{P}\left\{\log _{2}\left(1+\gamma_{1}\right)<\rho\right\}$ is the outage probability when $T=1$ given by Proposition 1 and the result follows.

We now derive the diversity order and coding gain of the selection-based scheme. Once again, we use the approximated expression in Theorem 2 for $T=1$, that is, $\Pi_{\mathrm{SB}}(\rho) \approx$ $\Pi_{\mathrm{CB}}^{\mathrm{CLT}}(\rho, 1)^{N}$. Hence, we have

$$
\lim _{P \rightarrow \infty}\left(\Pi_{\mathrm{CB}}^{\mathrm{CLT}}(\rho, 1)\right)^{N} \approx\left(\frac{\theta \sigma^{2}}{m P}\right)^{N} \rightarrow O\left(1 / P^{N}\right),
$$

which follows from $\exp (-x) \approx 1-x$ for $x \approx 0$. Thus, as expected, the selection-based scheme achieves full spatial diversity order with coding gain equal to

$$
G_{\mathrm{SB}}=\left(\frac{\sigma^{2}}{m}\right)^{N}\left(2^{\rho}-1\right)^{N}
$$

To compare $G_{\mathrm{SB}}$ with $G_{\mathrm{CB}}$, we need to consider the case $T=$ $N$, i.e. equal diversity order. If $m=M / N$, then it is clear that $G_{\mathrm{SB}}>G_{\mathrm{CB}}$. However, if each sub-surface employs $M$ elements then $G_{\mathrm{SB}}<G_{\mathrm{CB}}$; in this case, the selection-based scheme employs more elements ( $M N$ in total) but activates the same number as the coding-based scheme.

Similar to the coding-based scheme, in what follows, we evaluate the energy efficiency of the selection based scheme.

Proposition 4. The expected rate achieved by the selectionbased scheme is

$$
R_{\mathrm{SB}}=N \int_{0}^{\infty} \log _{2}\left(1+\frac{P}{\sigma^{2}} h\right) F_{H}(h)^{N-1} f_{H}(h) d h,
$$

where $f_{H}(h)$ and $F_{H}(h)$ are given by (10) and (24), respectively.

Proof. Given the SNR ordering $\gamma_{(1)} \geq \gamma_{(2)} \geq \cdots \geq \gamma_{(N)}$, the expected rate of the highest received SNR $\gamma_{(1)}$ at the destination can be derived as

$$
\mathbb{E}_{\gamma_{(1)}}\left\{\log _{2}\left(1+\gamma_{(1)}\right)\right\}=\mathbb{E}_{H_{1}}\left\{\log _{2}\left(1+\frac{P}{\sigma^{2}} H_{(1)}\right)\right\}
$$
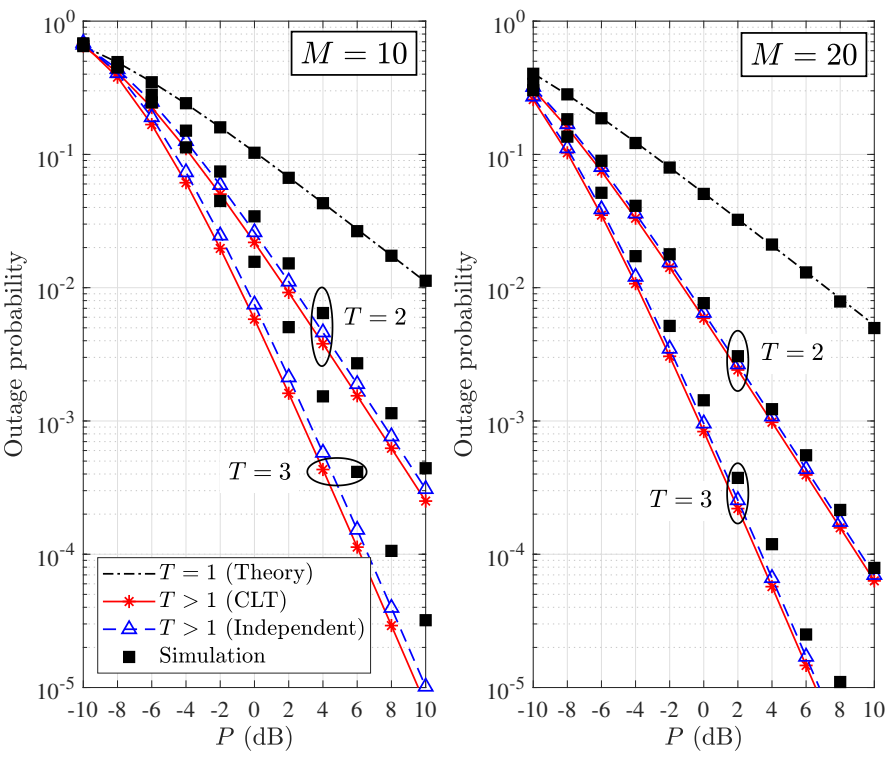

Fig. 2. Outage probability versus $P$ for the coding-based scheme.

$$
=\int_{0}^{\infty} \log _{2}\left(1+\frac{P}{\sigma^{2}} h\right) p_{H_{(1)}}(h) d h,
$$

where $p_{H_{(1)}}$ is the probability distribution function (PDF) of the largest order statistic $H_{(1)}$ given by [14]

$$
p_{H_{(1)}}(h)=N F_{H}(h)^{N-1} f_{H}(h) .
$$

By replacing the above PDF in (20), completes the proof.

Therefore, the energy efficiency achieved by the selectionbased scheme is

$$
\eta_{\mathrm{SB}}=\frac{R_{\mathrm{SB}}}{P / \xi+P_{S}+P_{D}+P_{\mathrm{IRS}}},
$$

where the power consumption parameters are defined as before but with $P_{\text {IRS }}=m P_{E}$, since only $m$ elements are activated at each time slot. It is clear, that $\eta_{\mathrm{SB}}=\eta_{\mathrm{CB}}$ when $N=T=1$. For $N=T>1$, the denominator of (22) is always less that the one of (15), since $m<M$.

\section{NumericAl Results}

In this section, we validate our theoretical analysis and main assumptions with computer simulations. For the sake of presentation, we consider $\rho=1 \mathrm{bps} / \mathrm{Hz}, \sigma^{2}=0 \mathrm{~dB}, \xi=1.2$, $P_{E}=10 \mathrm{dBm}, P_{D}=10 \mathrm{dBm}$ and $P_{S}=9 \mathrm{dBW}$ [6]. Unless otherwise stated, lines correspond to theoretical results whereas markers correspond to simulation results.

Fig. 2 depicts the outage probability achieved by the codingbased scheme in terms of the transmit power $P$, the number of channel uses $T$ and the number of reflecting elements $M$. As expected, the performance is improved with an increase of $M$. Moreover, and most importantly, increasing the number of channel uses $T$ provides significant gains to the outage probability. It should be highlighted that the massive benefits of this scheme are evident from $T=2$, which is the smallest 


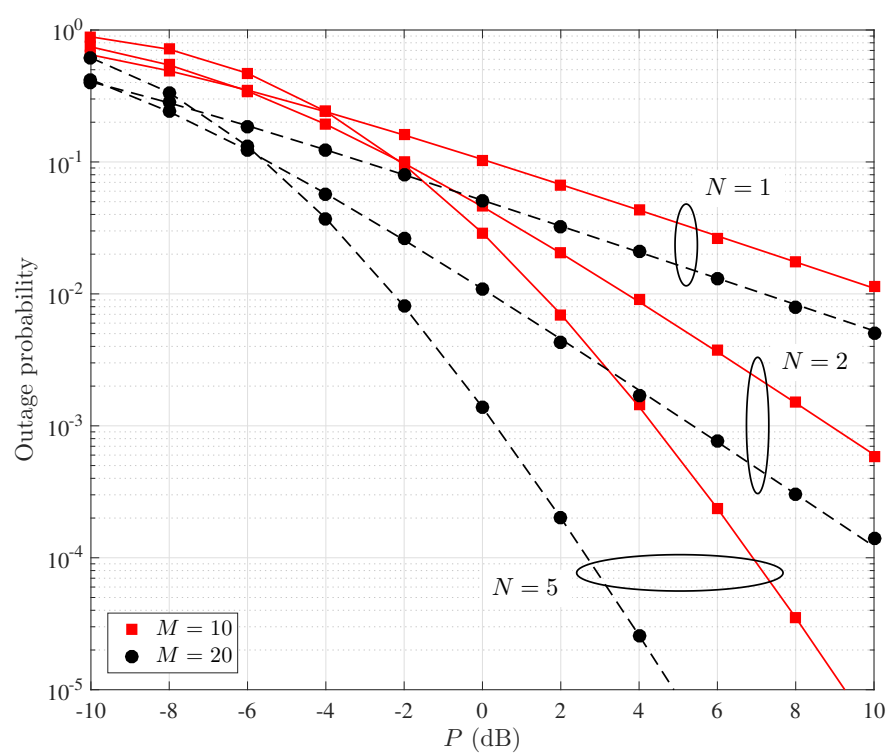

Fig. 3. Outage probability versus $P$ for the selection-based scheme.

number of channel uses the scheme can employ. Also, we can observe that the scheme provides full diversity order $T$, as deduced by our analysis. Finally, the figure validates the considered assumptions and approximations of our theoretical study. Specifically, for $T=1$, the simulation results perfectly match the theoretical result of Proposition 1. Furthermore, for $T>1$, the expressions of Theorem 1 and Theorem 2, approximate the achieved outage probability exceptionally well even for small $M$ and the approximations become tighter as $M$ increases.

Fig. 3 illustrates the performance of the selection-based scheme with regards to the number of reflecting elements $M$ and the number of sub-surfaces $N$. It is clear that the selection process improves the performance as $N$ increases, especially in the high SNR regime, where the scheme achieves full spatial diversity order. We should emphasize here that for $M=20$ and $N=5$, only four passive elements reflect their incident signal compared to the case $N=1$, for which there are twenty active elements. This shows how this scheme is energy efficient and of low-complexity but still provides performance gains. Again, the theoretical and simulation results are in agreement, which validates our analysis.

Finally, Fig. 4 shows the energy efficiency of the proposed schemes for different values of $M$ and $P$. The first main observation, is that the energy efficiency initially increases with $M$ but at some point it starts to decrease. This is expected, since more active elements implies higher rate but, at the same time, higher power consumption. Since the power consumption increases linearly, at some point, the rate-to-power-consumption ratio decreases. Secondly, the energy efficiency of the codingbased scheme is the same as with the conventional case $(T=1)$. This is because, on average, the rates of the two scenarios are equal. Finally, the selection-based protocol has a smaller energy efficiency for small values of $M$, compared to

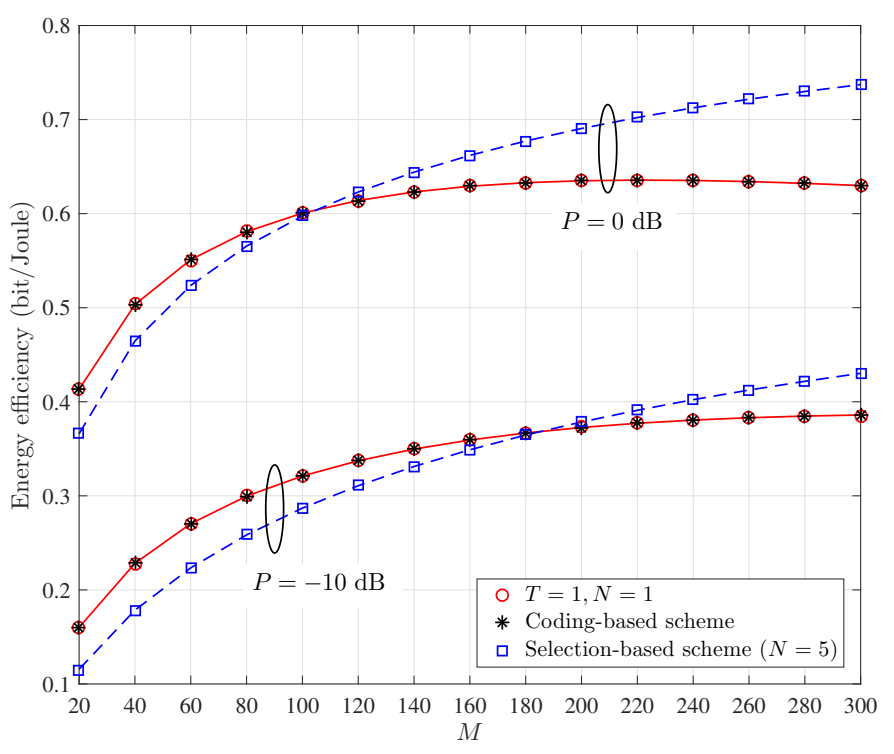

Fig. 4. Energy efficiency versus number of elements $M$.

the other two cases. However, as $M$ increases, the selectionbased scheme becomes more energy efficient due to the fact that it activates a fraction of the available elements at the IRS; this is clearly more evident for $P=0 \mathrm{~dB}$.

\section{CONCLUSion}

In this paper, we proposed two low-complexity and energy efficient schemes for IRS-aided communications via random phase rotations. In particular, we proposed a coding-based scheme, which produces a time-varying channel through timevarying random rotations. Moreover, we proposed a selectionbased scheme, which activates a partition of the IRS elements based on the highest received signal power. We derived analytical expressions for the outage probability and energy efficiency for both schemes. Our results demonstrated that the proposed schemes provide significant performance gains compared to the conventional case. Finally, we showed that both schemes exhibit full time (coding) and spatial (selection) diversity order. Future work includes the generalization of the considered model to multiple antennas and multiple users.

\section{APPENDIX A ProOF OF PROPOSITION 1}

Since $T=1$, the channel gain from the $M$ elements of the IRS is $H_{1}=\left|\sum_{i=1}^{M} h_{i} g_{i} \exp \left(j \phi_{1, i}\right)\right|^{2}$. Due to the random rotations, the phases have no effect on the channel gain. In other words, $H_{1}$ is statistically equivalent to $\left|\sum_{i=1}^{M} h_{i} g_{i}\right|^{2}$. Therefore, the outage probability when $T=1$ can be evaluated as follows

$$
\begin{aligned}
\Pi(\rho, 1) & =\mathbb{P}\left\{\log _{2}\left(1+\gamma_{1}\right)<\rho\right\}=\mathbb{P}\left\{\frac{P}{\sigma^{2}} H_{1}<2^{\rho}-1\right\} \\
& =\mathbb{P}\left\{\left|\sum_{i=1}^{M} h_{i} g_{i}\right|^{2}<\frac{\theta \sigma^{2}}{P}\right\},
\end{aligned}
$$




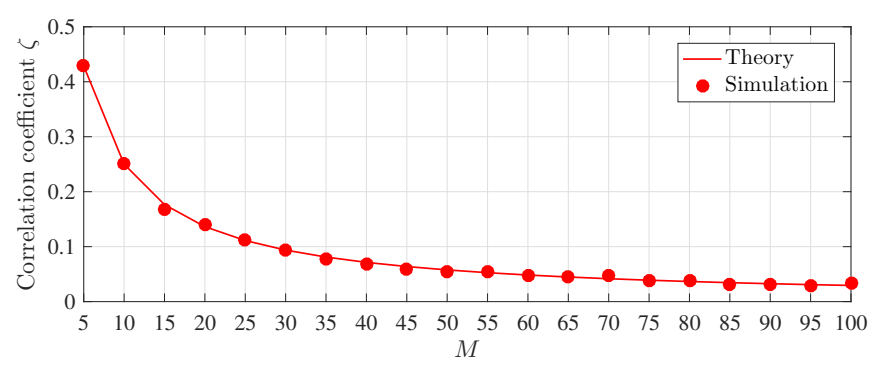

Fig. 5. Correlation coefficient $\zeta$ versus $M$.

where $\theta \triangleq 2^{\rho}-1$. The final expression is then derived by using the cumulative distribution function (CDF) of $H_{1}$, given by [15]

$$
F_{H_{1}}(x)=1-\frac{2 x^{M / 2} K_{M}(2 \sqrt{x})}{\Gamma(M)},
$$

where $K_{M}(\cdot)$ is the modified Bessel function of the second kind of order $M$ and $\Gamma(\cdot)$ is the complete gamma function.

\section{APPENDIX B}

\section{ProOf OF THEOREM 1}

The random variables $H_{t}=\left|\sum_{i=1}^{M} h_{i} g_{i} \exp \left(j \phi_{t, i}\right)\right|^{2}$ are correlated since the channel coefficients $h_{i}$ and $g_{i}$ remain constant over all $T$ transmissions. However, as $M$ increases, the correlation between the random variables $H_{t}$ decreases. In particular, the correlation coefficient $\zeta$ between $H_{i}$ and $H_{j}$, $i \neq j$, is given by

$$
\zeta=\frac{\mathbb{E}\left\{H_{i} H_{j}\right\}-\mathbb{E}\left\{H_{i}\right\} \mathbb{E}\left\{H_{j}\right\}}{\sigma_{H_{i}} \sigma_{H_{j}}}=\frac{3}{M+2},
$$

where it is clear that for $M \rightarrow \infty$ we have $\zeta \rightarrow 0$; this is also depicted in Fig. 5. By taking this into consideration, we can evaluate the outage probability as follows

$$
\begin{aligned}
& \Pi_{\mathrm{CB}}^{\mathrm{IND}}(\rho, T)=\mathbb{P}\left\{\frac{1}{T} \sum_{t=1}^{T} \log _{2}\left(1+\gamma_{t}\right)<\rho\right\} \\
& =\mathbb{P}\left\{\log _{2} \prod_{t=1}^{T}\left(1+\gamma_{t}\right)<T \rho\right\} \\
& =\mathbb{E}_{H_{t}}\left\{F_{H_{1}}\left(\frac{\sigma^{2}}{P}\left(\frac{2^{T \rho}}{\prod_{t=2}^{T}\left(1+P H_{t} / \sigma^{2}\right)}-1\right)\right)\right\},
\end{aligned}
$$

which follows by the logarithmic identity $\log _{2}(x)+\log _{2}(y)=$ $\log _{2}(x y)$, solving for $H_{1}$ and using the CDF of $H_{1}$ given by (24). Since the random variables $H_{t}$ are assumed to be independent, we have

$$
\begin{gathered}
\Pi_{\mathrm{CB}}^{\mathrm{IND}}(\rho, T)=\int_{z_{T}} \ldots \int_{z_{2}} F_{H_{1}}\left(\frac{\sigma^{2}}{P}\left(\frac{2^{T \rho}}{\prod_{t=2}^{T}\left(1+P z_{t} / \sigma^{2}\right)}-1\right)\right) \\
\times \prod_{t=2}^{T} f_{H_{t}}\left(z_{t}\right) d z_{2} \cdots d z_{T}
\end{gathered}
$$

where $f_{H_{t}}\left(z_{t}\right)$ is the PDF of $H_{t}$ given by the derivative of (24). The integration limits are evaluated by considering the inequality $\frac{2^{T \rho}}{\prod_{t=2}^{T}\left(1+P z_{t} / \sigma^{2}\right)}-1>0$ sequentially for each $z_{t}$. Finally, the transformation $w_{t} \rightarrow 1+P z_{t} / \sigma^{2}$ provides the final expression.

\section{APPENDIX C PROOF OF THEOREM 2}

Let $W_{t}=\sum_{i=1}^{M} h_{i} g_{i} \exp \left(j \phi_{t, i}\right)$. Then, $W_{t}$ can be expressed as $W_{t}=X_{t}+\jmath Y_{t}$ where $X_{t}=\sum_{i=1}^{M}\left|h_{i}\right|\left|g_{i}\right| \cos \left(\phi_{t, i}+\chi_{i}+\psi_{i}\right)$ and $Y_{t}=\jmath \sum_{i=1}^{M}\left|h_{i}\right|\left|g_{i}\right| \sin \left(\phi_{t, i}+\chi_{i}+\psi_{i}\right)$. By applying the CLT, we have that $X_{t}$ and $Y_{t}$ are Gaussian random variables with mean $\mu_{X_{t}}=\mu_{Y_{t}}=0$ and standard deviation $\sigma_{X_{t}}=$ $\sigma_{Y_{t}}=\sqrt{M / 2}$. As such, $W_{t}$ is a complex Gaussian random variable and thus $H_{t}=\left|W_{t}\right|^{2}$ is exponentially distributed with parameter $1 / M$. Since $\left|W_{t}\right|^{2}$ are independent of $t$, the final result can be derived by following similar steps to the proof of Theorem 1 with CDF $F_{H}(h)=1-\exp (-h / M)$ and PDF $f_{H}(h)=\exp (-h / M) / M$.

\section{REFERENCES}

[1] C. Liaskos, S. Nie, A. Tsioliaridou, A. Pitsillides, S. Ioannidis, and I. Akyildiz, "A new wireless communication paradigm through softwarecontrolled metasurfaces," IEEE Commun. Mag., vol. 56, no. 9, pp. 162 169, Sep. 2018.

[2] Q. Wu and R. Zhang, "Intelligent reflecting surface enhanced wireless network via joint active and passive beamforming," IEEE Trans. Wireless Commun., vol. 18, no. 11, pp. 5394-5409, Nov. 2019.

[3] Q. Nadeem, A. Kammoun, A. Chaaban, M. Debbah, M.-S. Alouini, "Asymptotic analysis of large intelligent surface assisted MIMO communication," [Online]. Available: https://arxiv.org/abs/1903.08127

[4] E. Basar, "Reconfigurable intelligent surface-based index modulation: A new beyond MIMO paradigm for 6G," IEEE Trans. Commun., vol. 68, no. 5, pp. 3187-3196, May 2020.

[5] Z. Ding and H. V. Poor, "A simple design of IRS-NOMA transmission," IEEE Commun. Lett., vol. 24, no. 5, pp. 1119-1123, May 2020.

[6] C. Huang, A. Zappone, G. C. Alexandropoulos, M. Debbah, and C. Yuen, "Reconfigurable intelligent surfaces for energy efficiency in wireless communication," IEEE Trans. Wireless Commun., vol. 18, no. 8, pp. 4157-4170, Aug. 2019.

[7] T. Hou, Y. Liu, Z. Song, X. Sun, Y. Chen, and L. Hanzo, "MIMO assisted networks relying on large intelligent surfaces: A stochastic geometry model," [Online]. Available: https://arxiv.org/abs/1910.00959

[8] Q. Wu and R. Zhang, "Weighted sum power maximization for intelligent reflecting surface aided SWIPT," IEEE Wireless Commun. Lett., vol. 9, no. 5, pp. 586-590, May 2020.

[9] C. Pan, H. Ren, K. Wang, M. Elkashlan, A. Nallanathan, J. Wang, and L. Hanzo, "Intelligent reflecting surface aided MIMO broadcasting for simultaneous wireless information and power transfer," [Online]. Available: https://arxiv.org/abs/1908.04863

[10] R. Pedarsani, O. Leveque, and S. Yang, "On the DMT optimality of timevarying distributed rotation over slow fading relay channels," IEEE Trans. Wireless Commun., vol. 14, no. 1, pp. 421-434, Jan. 2015.

[11] I. S. Gradshteyn and I. M. Ryzhik, Table of Integrals, Series, and Products. Elsevier, 2007.

[12] M. Uysal, "Diversity analysis of space-time coding in cascaded Rayleigh fading channels," IEEE Commun. Lett., vol. 10, no. 3, pp. 165-167, Mar. 2006.

[13] D. Tse and P. Viswanath, Fundamentals of Wireless Communication. Cambridge University Press, 2005.

[14] H. Yang and M.-S. Alouini, Order Statistics in Wireless Communications: Diversity, Adaptation, and Scheduling in MIMO and OFDM Systems. Cambridge University Press, 2011.

[15] J. D. Griffin and G. D. Durgin, "Gains for RF tags using multiple antennas," IEEE Trans. Antennas Propagat., vol. 56, no. 2, pp. 563-570, Feb. 2008. 